Качество жизни детей с болезнью Крона как потенциальный критерий мониторинга активности заболевания

\author{
А.Р. Тагирова, И.В. Сичинава, О.А. Савватеева, Е.В. Борисова \\ ФГАОУ ВО «Первый Московский государственный медицинский университет имени И.М. Сеченова» Министерства здравоохранения \\ Российской Федерации (Сеченовский Университет); Россия, г. Москва
}

\title{
PЕЗЮМЕ
}

Цель исследования: анализ динамики показателей активности болезни Крона (БК) и качества жизни (КЖ) детей 8-17 лет. Дизайн: открытое проспективное нерандомизированное сравнительное исследование.

Материалы и методы. Выполнена оценка КЖ у 37 практически здоровых детей и 28 детей с БК (17 мальчиков и 11 девочек). Активность заболевания (индекс Pediatric Crohn's Disease Activity Index, PCDAI) определяли по опроснику PedsQL 4.0 Generic Core Scales, который включает оценку состояния здоровья и активности, настроения детей, сложностей в общении, особенностей обучения в школе. Наблюдение проводили в течение 18 месяцев, исследуемые показатели оценивали в начале наблюдения (при первом обследовании), через 6, 12 и 18 месяцев. КЖ оценивали как сами дети, так и их родители, показатели были оценены в динамике и сопоставлены с активностью, длительностью заболевания, а также с наличием внекишечных проявлений болезни.

Результаты. У детей с БК 8-17 лет отмечается снижение показателей большинства шкал опросника PedsQL 4.0. Bыявленные сдвиги наблюдаются и при оценке КЖ детьми, и по результатам опроса их родителей. Лечение этих пациентов сопровождается положительной динамикой КЖ, и данные изменения происходят параллельно со снижением индекса активности БК, что позволяет предположить возможность использования параметров КЖ для прогноза течения болезни и эффективности проводимого лечения у данного контингента больных.

Значения индекса PCDAI у обследуемых детей имели обратные статистически значимые корреляции умеренной силы с параметрами шкал «Физическая активность» $(r=-0,412)$, «Настроение» $(r=-0,364)$, «Психосоциальное функционирование» $(r=-0,306)$ и «Жизнь в школе» $(r=-0,344)$ и с общим показателем опросника PedsQL $4.0(r=-0,406)$. Длительность заболевания статистически значимо отрицательно связана с показателями шкал «Физическая активность» $(r=-0,386)$, «Настроение» $(r=-0,423)$, «Психосоциальное функционирование» $(r=-0.345)$, с интегральным показателем опросника PedsQL $4.0(r=-0,397)$, а наличие внекишечных проявлений заболевания умеренно отрицательно коррелировало со значениями шкал «Физическая активность» $(r=-0,342)$, «Жизнь в школе» $(r=-0,431)$ и общим показателем $(r=-0,372)$.

Заключение. Обязательным компонентом обследования и ведения детей с воспалительными заболеваниями кишечника должен стать мониторинг уровня КЖ, связанного со здоровьем, поскольку для этой категории больных характерно значительное его снижение относительно соответствующих показателей у здоровых сверстников.

Ключевые слова: воспалительные заболевания кишечника, болезнь Крона, качество жизни, активность заболевания, внекишечные проявления.

Вклад авторов: Сичинава И.В. - создание концепции, постановка задачи исследования, анализ результатов и подготовка данных, написание текста статьи; Тагирова А.Р. - выполнение рутинной работы по систематизации материала, анализ результатов исследования и подготовка данных, написание текста статьи; Савватеева 0.А., Борисова Е.В. - ведение пациентов и сбор материала для статьи.

Конфликт интересов: авторы заявляют об отсутствии возможных конфликтов интересов.

Для цитирования: Тагирова А.Р., Сичинава И.В., Савватеева О.А., Борисова Е.В. Качество жизни детей с болезнью Крона как потенциальный критерий мониторинга активности заболевания. Доктор.Ру. 2020; 19(10): 27-32. DOI: 10.31550/1727-2378-2020-19-10-27-32

\section{Quality of Life of Children with Crohn's Disease as a Potential Criterion for Monitoring Disease Activity}

\author{
A.R. Tahirova, I.V. Sichinava, O.F. Savvateeva, E.V. Borisova \\ I.M. Sechenov First Moscow State Medical University (Sechenov University) (a Federal Government Autonomous Educational Institution of \\ Higher Education), Russian Federation Ministry of Health; 19 Bolshaya Pirogovskaya Str., Bldg. 1, Moscow, Russian Federation 119991
}

\section{ABSTRACT}

Study Objective: To analyse the activity dynamics of Crohn's disease (CD) and the quality of life ( $Q 0 \mathrm{~L})$ of children aged 8-17 years.

Study Design: Open prospective non-randomized comparative study.

Materials and Methods. The quality of life was assessed in 37 healthy children and 28 children with Crohn's disease (17 boys and 11 girls). Disease activity (Paediatric Crohn's Disease Activity Index, PCDAI) was determined using PedsQL 4.0 Generic Core Scales questionnaire which

Тагирова Аният Руфатовна (автор для переписки) - аспирант кафедры детских болезней Клинического института детского здоровья имени Н.Ф. Филатова ФГАОУ ВО «Первый МГмУ им. И.М Сеченова» Минздрава России (Сеченовский Университет). 119991, Россия, г. Москва, ул. Большая Пироговская, д. 19, cmp. 1. http://orcid.org/0000-0002-4809-2405.Email: aniyat.tahirova@mail.ru

Сичинава Ирина Вениаминовна - д. м. н., профессор кафедры детских болезней Клинического института детского здоровья имени Н.Ф. Филатова ФГАОУ ВО «Первый МГмУ им. И.М Сеченова» Минздрава России (Сеченовский Университет). 119991, Россия, г. Москва, ул. Большая Пироговская, д. 19, cmp. 1. http://orcid.org/0000-0002-6140-504X. Email: sichinava@mail.ru

(Окончание на С. 28.) 
comprises assessment of health and activity, child's attitude, problems with interpersonal relations, and progress at school. Observation spanned over 18 months; tests parameters were assessed at the beginning (first visit), after 6,12 and 18 months. QoL was assessed by both children and their parents, the indicators were assessed over time and compared with the levels of activity, the duration of the disease, and the presence of extraintestinal manifestations of the disease.

Study Results. Children with CD aged 8-17 years demonstrated a decrease in the majority of PedsQL 4.0 parameters. The shifts are observed both when $\mathrm{QoL}$ is assessed by children and their parents. The therapy in these patients is accompanied by positive QoL dynamics; these changes occur in parallel with a decrease in the values of the CD activity index, suggesting the possibility of using the QoL parameters to predict the course of the disease and the effectiveness of the treatment in this population.

PCDAI values in subjects demonstrated moderate statistically significant negative correlations with "Physical activity" $(r=-0.412)$, "Attitude" ( $r=-0.364)$, "Psychosocial functioning" $(r=-0.306)$ and "School life" $(r=-0.344)$ and the overall PedsQL 4.0 values $(r=-0.406)$. The duration of the disease has statistically significant negative correlation with "Physical activity" $(r=-0.386)$, "Attitude" $(r=-0.423)$, "Psychosocial functioning" ( $r=-0.345)$, and the integral PedsQL 4.0 value $(r=-0.397)$; the presence of extraintestinal manifestations demonstrated moderate negative correlation with "Physical activity" $(r=-0.342)$, "School life" $(r=-0.431)$ and overall value $(r=-0.372)$. Conclusion. Monitoring of the health-related quality of life should become a mandatory component of the examination and management of children with inflammatory bowel diseases, since this category of patients is characterized by a significant decrease relative to the corresponding indicators in healthy peers.

Keywords: inflammatory bowel disorder, Crohn's disease, quality of life, disease activity, extraintestinal manifestations.

Contributions: Sichinava, I.V. - concept, study objectives, results analysis and data preparation, text of the article; Tahirova, A.R. - routine material systematisation, study results analysis and data preparation, text of the article; Savvateeva, 0.F. and Borisova, E.V. - management of the patient and collection of materials of the article.

Conflict of interest: The authors declare that they do not have any conflict of interests.

For citation: Tahirova A.R., Sichinava I.V., Savvateeva 0.F., Borisova E.V. Quality of Life of Children with Crohn's Disease as a Potential Criterion for Monitoring Disease Activity. Doctor.Ru. 2020; 19(10): 27-32. (in Russian). D0I: 10.31550/1727-2378-2020-19-10-27-32

\section{ВВЕДЕНИЕ}

Воспалительные заболевания кишечника (ВЗК) - группа хронических заболеваний (болезнь Крона (БК) и язвенный колит), характеризующихся воспалением слизистой оболочки ЖКТ, которое проявляется периодами обострения и ремиссии $[1,2]$. Распространенность и заболеваемость ВЗК во всем мире растет, в последние годы ее уровень составляет от 4 до 11 случаев на 100000 населения [3]. В возрасте до 20 лет приблизительно у 25-30\% пациентов выявляется БК, у 20-25\% - язвенный колит [2, 3].

Более чем у 75\% детей с БК отмечаются нарушения роста и недостаток массы тела $[4,5]$. Причинами этих нарушений являются анорексия, мальабсорбция, воспаление в кишечнике, а также проводимое медикаментозное лечение. Наблюдается снижение физической активности детей с БК, которое, как полагают, связано в значительной мере с уменьшением мышечной массы [6].

На сегодняшний день выполнен ряд исследований, посвященных оценке качества жизни (КЖ) пациентов с ВЗК, и согласно их результатам, у большинства больных показатели КЖ значимо снижены по сравнению с соответствующими характеристиками в общей популяции $[2,4,7,8]$.

Показано, что для детей и подростков с ВЗК, в том числе с БК, характерны нарушения взаимоотношений в семье [9], ограниченное участие в общественной деятельности, расстройства эмоционального спектра, проблемы в учебе, более частые пропуски занятий $[8,9]$. Имеются сообщения о более высокой частоте психических расстройств, особенно тревожности и депрессии, у детей, страдающих ВЗК, по сравнению с таковой среди здоровых детей [10], у этой категории пациентов снижена самооценка, отмечаются сложности социального взаимодействия с другими детьми [11].
В качестве одного из наиболее удобных и надежных инструментов оценки КЖ детей, страдающих различными заболеваниями, в настоящее время рассматривается опросник PedsQL 4.0 Generic Core Scales, перевод которого на русский язык признан валидным [12]. Однако следует отметить, что сообщения об оценке КЖ в педиатрической популяции больных БК, в том числе с применением данного опросника, в доступной литературе единичны. Недостаточно изучены взаимосвязи нарушений КЖ у таких больных с клиническими проявлениями и уровнем активности заболевания.

Цель исследования: анализ динамики показателей активности БК и КЖ у детей 8-17 лет.

\section{МАТЕРИАЛЫ И МЕТОДЫ}

В 2017-2020 гг. на базе Университетской детской клинической больницы и кафедры детских болезней Клинического института детского здоровья ФГАОУ В0 «Первый МГМУ имени И.М. Сеченова» МЗ РФ (Сеченовский Университет) проведено открытое проспективное нерандомизированное сравнительное исследование: выполнена оценка КЖ у 37 практически здоровых детей (группа 1, контрольная) и 28 детей с БК (17 мальчиков и 11 девочек), которые составили группу 2. Средний возраст детей, включенных в исследование, $-12,5 \pm 4,2$ года (от 8 до 17 лет).

Диагноз у детей с БК был поставлен на основании комплексного обследования, включающего клинико-лабораторные данные и результаты эндоскопического обследования на основании рекомендаций по диагностике и лечению БК у детей (2016) [13].

При этом в исследование не включали детей, у которых была достигнута стойкая ремиссия основного заболевания на момент начала исследования, а также при наличии

Савватеева Ольга Александровна - аспирант кафедры детских болезней Клинического института детского здоровья имени Н.Ф. Филатова ФГАОУ ВО «Первый МГмУ им. И.М Сеченова» Минздрава России (Сеченовский Университет). 119991, Россия, г. Москва, ул. Большая Пироговская, Ә. 19, стр. 1. eLIBRARY.RU SPIN:1052-7020. http://orcid.org/0000-0003-4919-9332. E-mail: olya-benda@mail.ru

Борисова Елена Васильевна - к. м. н., заведующая гастроэнтерологическим отделением Клинического института детского здоровья имени Н.Ф. Филатова ФГАОУ ВО «Первый МГмУ им. И.М Сеченова» Минздрава России (Сеченовский Университет). 119991, Россия, г. Москва, ул. Большая Пироговская, д. 19, стр. 1. http://orcid.org/0000-0003-4893-6185. E-mail: bor-lena@yandex.ru (Окончание. Начало см. на с. 27.) 
сопутствующих заболеваний, которые могли бы повлиять на течение основного заболевания, исказить клиническую картину и результаты лабораторно-инструментальной диагностики.

Активность БК оценивали с применением клинико-лабораторного индекса Pediatric Crohn's Disease Activity Index (PCDAI) [13].

Средняя длительность заболевания у детей с БК составила $5,7(2,9 ; 8,5)$ года. В процессе наблюдения осуществляли сравнительную оценку показателей КЖ детей с помощью опросника PedsQL 4.0 Generic Core Scales для возрастной группы 8-17 лет, шкалы которого предусматривают возможность оценить уровень КЖ ребенка по следующим составляющим: физическая активность, настроение, психосоциальное функционирование, общение, жизнь в школе. Пациентам предлагалось оценить ситуации, в которых они испытывали трудности в течение последнего месяца. При этом КЖ оценивали самостоятельно как дети, так и родители.

Параметры КЖ были определены в динамике и сопоставлены с активностью, длительностью заболевания, а также наличием внекишечных проявлений болезни.

Лечение всех детей с БК проводили в соответствии с клиническими рекомендациями [13]. Терапия была направлена на индукцию ремиссии, профилактику рецидивов, предупреждение прогрессирования и развития осложнений, в том числе требующих хирургического вмешательства.

С целью индукции ремиссии в качестве препаратов первой линии в зависимости от тяжести атаки и наличия внекишечных проявлений назначали системные и/или топические ГКС - препараты преднизолона. Дозы рассчитывали на основании массы тела детей; они, как правило, составляли до 40 мг/сут с постепенным снижением по 10 мг/нед в виде монотерапии либо в комбинации с иммуносупрессивными лекарственными средствами - азатиоприном в дозе 2-2,5 мг/кг/сут, 6-меркаптопурином в дозе 1,5 мг/кг/сут или метотрексатом в дозе до 15 мг/м²/нед [13].

При неэффективности или плохой переносимости терапии первой линии к лечению по показаниям добавляли иммуносупрессорные и/или генно-инженерные биологические препараты (инфликсимаб и адалимумаб). Дозу препарата определяли на основании массы тела ребенка.

Наблюдение за пациентами, включенными в исследование, проводили в течение 18 месяцев, исследуемые показатели оценивали в начале наблюдения (при первом обследовании), через 6, 12 и 18 месяцев.

Анализ результатов исследования выполнен с помощью пакетов программного обеспечения Statsoft Statistica 10 и Microsoft Excel 2016. Выбор основных характеристик и статистических критериев при их сравнении осуществляли после изучения распределения признака и его сравнения с распределением Гаусса по критерию Колмогорова - Смирнова. Поскольку выявленное распределение признаков отличалось от нормального, для дальнейшей работы с полученными данными использовали непараметрические методы.

Количественные данные описаны в виде $\mathrm{Me}\left(Q_{25} ; Q_{75}\right)$, где $\mathrm{Me}$ - медиана; $Q_{25}$ и $Q_{75}$ - нижний и верхний квартили. Качественные параметры представлены в виде частот встречаемости признаков в процентах от общего числа пациентов. Значения количественных показателей в группах больных (несвязанные выборки) сравнивали с использованием критерия Манна - Уитни.

Поиск взаимосвязей характеристик КЖ с показателями активности заболевания проводили с помощью корреляционного анализа с расчетом коэффициента корреляции Спирмена.
Различия считались статистически значимыми при недостижении р порогового значения уровня статистической значимости нулевой гипотезы (альфа), равного 0,05.

\section{РЕЗУЛЬТАТЬ}

Анализ основных клинических проявлений заболевания у детей с БК показал, что наиболее частыми симптомами были боль в животе и болезненность при пальпации живота. У некоторых детей наблюдались внекишечные проявления заболевания: отмечено 2 (7,1\%) случая первичного склерозирующего холангита, у 7 (25,0\%) больных - поражения суставов.

Изучение активности заболевания у пациентов с БК свидетельствовало о том, что до начала исследования величина индекса PCDAI составила $38,8(34,7 ; 41,7)$ балла. В процессе наблюдения отмечалось клинически значимое снижение активности БК: через 6 мес - до $22,5(18,6 ; 25,8)$ балла. При этом значение данного показателя было статистически значимо $(p=0,043)$ ниже исходного уровня.

Спустя год после начала наблюдения индекс PCDAI снизился до $14,9(12,9 ; 18,3)$ балла, а через 18 мес - до 11,2 $(9,3 ; 15,9)$ балла, что было статистически значимо меньше значения до начала наблюдения, а также показателя через 6 мес ( $p=0,018$ и $p=0,004$ соответственно).

Результаты исходных оценок уровня КЖ детей (при первичном обследовании) приведены в таблице 1. Отмечено существенное снижение $(p<0,05)$ показателей КЖ детей с БК по всем шкалам опросника PedsQL 4.0 Generic Core Scales при оценке как детьми, так и их родителями. Значения всех шкал у пациентов были статистически значимо ниже $(p<0,05)$ таковых в контрольной группе. При этом оценки, данные родителями, были несколько ниже оценок самих детей, но статистически значимые различия между этими значениями отсутствовали.

Изучение динамики показателей КЖ детей, включенных в исследование, свидетельствовало о постепенном повышении параметров PedsQL 4.0 Generic Core Scales и по оценкам больных БК, и согласно результатам опроса их родителей. При этом первым реагировал показатель шкалы «Настроение».

При оценке детьми значение общего показателя опросника через 6 мес от начала наблюдения несколько увеличилось, хотя в этот срок статистически значимые отличия от исходного уровня не отмечены. Через 12 мес значение данного параметра КЖ составило 69,1 (62,0; 70,2) балла, спустя 18 мес оно возросло до 70,8 $(62,4 ; 81,5)$ балла, оба значения статистически значимо превышали исходный показатель (соответственно $\mathrm{p}=0,016$ и $\mathrm{p}=0,011$ ).

Анализ оценок КЖ детей с БК, которые были даны их родителями, также показал постепенное возрастание общего показателя опросника, при этом значение данного параметра статистически значимо ( $p=0,020)$ увеличилось от 60,8 (51,3; 74,8) (исходно) до $63,2(56,3 ; 74,0)$ балла через 6 мес после начала наблюдения. Спустя год величина параметра достигла 69,1 $(62,4 ; 78,4)$ балла, а через 18 мес оставалась практически на том же уровне - 70,3 $(62,2 ; 81,4)$ балла (табл. 2).

При этом показатели шкал опросника «Психосоциальное функционирование», «0бщение» и «Жизнь в школе» не отличались от соответствующих параметров у здоровых детей, в то время как значения шкал «Физическая активность», «Настроение» и общий показатель опросника были статистически значимо ниже $(p<0,05)$, чем в контрольной группе. 
Таблица 1 / Table 1

Показатели опросника PedsQL 4.0 Generic Core Scales пациентов с болезнью Крона (БК) до начаца набцюдения, бамлы, $\mathrm{Me}\left(Q_{25} ; Q_{75}\right)$

PedsQL 4.0 Generic Core Scales values in patients with Crohn's disease (CD) prior to observation, points, $\mathrm{Me}\left(\mathrm{Q}_{25} ; \mathrm{Q}_{75}\right)$

\begin{tabular}{|c|c|c|c|}
\hline $\begin{array}{c}\text { Шкалы PedsQL 4.0 Generic Core Scales / } \\
\text { PedsQL 4.0 Generic Core Scales }\end{array}$ & $\begin{array}{c}\text { Группа } 1 \\
\text { (контрольная) / } \\
\text { Group } 1 \text { (Control) } \\
\quad(\mathrm{n}=37)\end{array}$ & $\begin{array}{c}\text { Группа } 2 \text { (дети с БК) / } \\
\text { Group } 2 \text { (Children with CD) } \\
(\mathbf{n}=28)\end{array}$ & $\begin{array}{l}\text { Оценка родителями } \\
\text { детей с БК / Assessment } \\
\text { of children with CD by } \\
\text { parents }(n=28)\end{array}$ \\
\hline Общий показатель / Overall value & $83,5(71,3 ; 94,2)$ & $64,1(56,7 ; 76,1)^{*}$ & $60,8(51,3 ; 74,8)$ \\
\hline $\begin{array}{l}\text { Психосоциальное функционирование / } \\
\text { Psychosocial functioning }\end{array}$ & $82,9(72,6 ; 93,0)$ & $67,3(58,2 ; 80,9)^{*}$ & $57,5(48,1 ; 69,4)$ \\
\hline Физическая активность / Physical activity & $91,9(78,5 ; 98,2)$ & $66,7(55,9 ; 81,4)^{*}$ & $60,7(52,0 ; 74,9)$ \\
\hline Настроение / Attitude & $78,2(65,4 ; 90,7)$ & $62,1(51,0 ; 75,2)^{*}$ & $52,9(41,3 ; 67,5)$ \\
\hline Общение / Interpersonal relations & $88,5(80,3 ; 95,9)$ & $79,3(69,2 ; 89,3) *$ & $71,2(60,2 ; 84,9)$ \\
\hline Жизнь в школе / School life & $76,9(66,3 ; 87,6)$ & $61,4(50,8 ; 74,5)^{*}$ & $56,1(43,7 ; 72,5)$ \\
\hline
\end{tabular}

* 3Аесь и в таблице 2: отАичия от групшы 1 (контрольной) статистически значимы по критерию Манна Уитни $(\mathrm{p}<0,05)$.

* Here and table 2: differences vs. group 1 (controls) are statistically significant, Mann-Whitney test $(\mathrm{p}<0.05)$.

Таблица 2 / Table 2

Показатели опросника PedsQL 4.0 Generic Core Scales пациентов с болезнью Крона (БК) через 18 месяцев после начала наблюдения, бамлы, $\mathrm{Me}\left(\mathrm{Q}_{25} ; \mathrm{Q}_{75}\right)$

PedsQL 4.0 Generic Core Scales values in patients with Crohn's disease (CD) after 18 months of observation, points, $\mathrm{Me}\left(\mathrm{Q}_{25} ; \mathrm{Q}_{75}\right)$

\begin{tabular}{|c|c|c|c|}
\hline $\begin{array}{c}\text { Шкалы PedsQL 4.0 Generic Core Scales / } \\
\text { PedsQL 4.0 Generic Core Scales }\end{array}$ & $\begin{array}{c}\text { Группа } 1 \\
\text { (контрольная) / } \\
\text { Group } 1 \text { (Control) } \\
\quad(\mathrm{n}=37)\end{array}$ & $\begin{array}{c}\text { Группа } 2 \text { (дети с БК) / } \\
\text { Group } 2 \text { (Children with CD) } \\
(n=28)\end{array}$ & $\begin{array}{c}\text { Оценка родителями } \\
\text { детей с БК/ Assessment } \\
\text { of children with CD by } \\
\text { parents } \\
(\mathrm{n}=28)\end{array}$ \\
\hline Общий показатель / Overall value & $83,5(71,3 ; 94,2)$ & $70,8(62,4 ; 81,5)^{*}$ & $70,3(62,2 ; 81,4)$ \\
\hline $\begin{array}{l}\text { Психосоциальное функционирование / } \\
\text { Psychosocial functioning }\end{array}$ & $82,9(72,6 ; 93,0)$ & $78,2(65,1 ; 84,4)$ & $74,0(63,3 ; 78,4)$ \\
\hline Физическая активность / Physical activity & $91,9(78,5 ; 98,2)$ & $77,2(62,7 ; 85,9)^{*}$ & $69,3(60,3 ; 80,5)$ \\
\hline Настроение / Attitude & $78,2(65,4 ; 90,7)$ & $68,4(60,2 ; 78,2)^{*}$ & $64,0(58,4 ; 78,1)$ \\
\hline Общение / Interpersonal relations & $88,5(80,3 ; 95,9)$ & $87,2(75,2 ; 96,5)$ & $88,1(72,8 ; 94,7)$ \\
\hline Жизнь в школе / School life & $76,9(66,3 ; 87,6)$ & $70,7(61,5 ; 82,8)$ & $68,0(58,5 ; 78,2)$ \\
\hline
\end{tabular}

Поиск взаимосвязей показателей КЖ с характеристиками клинической активности БК с помощью корреляционного анализа показал, что значения индекса PCDAI у обследуемых детей имели обратные статистически значимые корреляции умеренной силы с параметрами шкал «Физическая активность» ( $r=-0,412)$, «Настроение» $(r=-0,364)$, «Психосоциальное функционирование» $(r=-0,306)$ и «Жизнь В школе» $(r=-0,344)$ (табл. 3). В то же время значимых корреляций для шкалы «Общение» с уровнем активности заболевания не было. При этом величина индекса PCDAI также отрицательно статистически значимо ассоциирована с общим показателем опросника PedsQL $4.0(r=-0,406)$.

Установлено, что длительность заболевания значимо отрицательно связана с показателями шкал «Физическая активность» $(r=-0,386)$, «Настроение» $(r=-0,423)$, «Психосоциальное функционирование» $(r=-0,345)$, а также интегральным показателем опросника PedsQL 4.0 ( $r=-0,397)$.

Наличие внекишечных проявлений заболевания умеренно отрицательно коррелировало со значениями шкал
«Физическая активность» $(r=-0,342)$, «Жизнь в школе» $(r=-0,431)$ и общим показателем опросника $(r=-0,372)$.

\section{ОБСУЖДЕНИЕ}

Проведенное изучение уровня КЖ у пациентов, включенных в исследование, позволило выявить снижение показателей различных шкал опросника PedsQL 4.0 при оценке как самими детьми с БК, так и их родителями. Результаты корреляционного анализа подтвердили наличие ряда статистически значимых отрицательных взаимосвязей умеренной силы между клиническими характеристиками заболевания, с одной стороны, и показателями качества жизни детей с БК - с другой. Значения ряда шкал опросника PedsQL 4.0 Generic Core Scales значимо связаны с индексом активности БК, а также с длительностью болезни и наличием внекишечных проявлений.

Следует отметить, что в настоящее время механизмы, лежащие в основе взаимосвязей изменений КЖ у детей с ВЗК и параметров активности заболевания, до настоящего времени изучены недостаточно. Z. Ojeda, C. Cofré (2018) 
Таблица 3 / Table 3

Взаимосвязь показателей качества жизни с активностью, Алительностью заболевания и наличием внекишечных проявлений у пациентов с болезнью Крона

(коэффициенты корремяции Спирмена)

Interrelation between quality of life and activity, duration of disease and presence of extraintestinal manifestations in patients with Crohn's disease (Spearman correlation coefficients)

\begin{tabular}{|l|l|l|l|}
\hline \multicolumn{1}{|c|}{$\begin{array}{c}\text { Шкалы PedsQL 4.0 Generic Core } \\
\text { Scales / PedsQL 4.0 Generic Core Scales }\end{array}$} & $\begin{array}{c}\text { Pediatric Crohn's } \\
\text { Disease Activity Index }\end{array}$ & $\begin{array}{c}\text { Длительность } \\
\text { заболевания / Duration } \\
\text { of disease }\end{array}$ & $\begin{array}{c}\text { Наличие внекишечных } \\
\text { проявлений заболевания / } \\
\text { Presence of extraintestinal } \\
\text { manifestations }\end{array}$ \\
\hline $\begin{array}{l}\text { Психосоциальное функционирование / } \\
\text { Psусhоsосіal functioning }\end{array}$ & $-0,306(p=0,045)$ & $-0,345(p=0,029)$ & $-0,288(p=0,082)$ \\
\hline $\begin{array}{l}\text { Физическая активность / Physical } \\
\text { астіvity }\end{array}$ & $-0,412(p=0,011)$ & $-0,386(p<0,001)$ & $-0,342(p=0,021)$ \\
\hline Настроение/ Attitude & $-0,364(p=0,025)$ & $-0,423(p=0,009)$ & $-0,263(p=0,132)$ \\
\hline Общение / Interpersonal relations & $-0,229(p=0,018)$ & $-0,205(p=0,176)$ & $-0,187(p=0,128)$ \\
\hline Жизнь в школе / School life & $-0,344(p=0,003)$ & $-0,218(p=0,212)$ & $-0,431(p=0,005)$ \\
\hline Общий показатель / Overall value & $-0,406(p<0,001)$ & $-0,397(p=0,023)$ & $-0,372(p<0,001)$ \\
\hline
\end{tabular}

предложили рассматривать активность заболевания как фактор, связанный с КЖ. По мнению авторов, понимание того, какие патогенетические и клинические факторы у больных ВЗК значимо связаны с уровнем КЖ, позволит обосновать необходимость выполнения тех или иных лечебных мероприятий. Это, в свою очередь, поможет повысить КЖ данного контингента больных [14].

Ремитирующий характер заболевания и частые его рецидивы у педиатрических больных с ВЗК оказывают значительное воздействие на КЖ. Поиск ассоциаций между клиническими проявлениями заболевания и показателями КЖ у детей показал, что значения большинства шкал опросника коррелируют с основными клиническими характеристиками течения БК. Полученные данные согласуются с результатами других авторов. Так, М. van der Have и соавт. (2015) выявили взаимосвязь между внекишечными проявлениями заболевания и эмоциональным состоянием больных. Показано, что для пациентов, длительно страдающих В3К, характерен более низкий уровень КЖ [15].

В нашем исследовании установлено, что длительность заболевания статистически значимо отрицательно связана в большей степени с показателями шкал «Физическая активность» и «Настроение», а также общим показателем опросника. С большинством шкал PedsQL 4.0 также связаны активность заболевания (индекс PCDAI) и наличие внекишечных проявлений ВЗК.

Проведенное нами исследование показало, что оценки КЖ, данные родителями обследуемых детей, были несколько ниже оценок самих детей, но статистически значимых различий между ними не было. Важную роль в успешном ведении детей с ВЗК отводят родителям М. Bramuzzo и соавт. (2019). По их мнению, изменения психологического благополучия родителей могут повлиять на уровень КЖ этих пациентов. Авторы продемонстрировали, что выраженность родительского дистресса имела наиболее существенную корреляцию с уровнем КЖ (по Health-related quality of life) у детей,

\section{ЛИТЕРАTУPA / REFERENCES}

1. CarrollM.W., Kuenzig M.E.,Mack D.R. et al. The impact of inflammatory bowel disease in Canada 2018: children and adolescents with IBD. J. Can. Assoc. Gastroenterol. 2019; 2(suppl.1): S49-67. DOI: 10.1093/jcag/gwy056

2. Knowles S.R., Keefer L., Wilding H. et al. Quality of life in inflammatory bowel disease: a systematic review and meta- а также была связана с активностью болезни и недавними обострениями ВЗК. При многофакторном регрессионном анализе установлено, что вклад «родительских факторов» в дисперсию показателей КЖ у детей составил не более 20\% [16].

По нашему мнению, следует согласиться с выводом авторов о необходимости осуществления мероприятий, направленных на коррекцию родительского дистресса, при ведении детей с ВЗК.

Полученные нами данные свидетельствуют о том, что в ходе обследования детей с ВЗК педиатр должен оценивать наличие социально-психологических стрессовых факторов у каждого ребенка, опрашивать родителей об успеваемости и посещаемости школы детьми.

\section{ЗАКЛЮЧЕНИЕ}

Полученные нами результаты говорят о том, что у детей с воспалительными заболеваниями кишечника (ВЗК) 8-17 лет отмечается снижение качества жизни (КЖ), согласно результатам обследования с применением опросника PedsQL 4.0 Generic Core Scales. Наиболее значимые изменения касаются физической активности, настроения и психосоциального функционирования.

Выявленные сдвиги наблюдаются и при оценке КЖ детьми с болезнью Крона (БК), и при опросе их родителей. Лечение пациентов сопровождается положительной динамикой уровня КЖ, при этом первым реагирует показатель настроения детей. Указанные изменения происходят параллельно со снижением индекса активности БК, что позволяет предположить возможность использования параметров КЖ для прогноза течения болезни и эффективности проводимого лечения у данного контингента больных.

Обязательным компонентом обследования и ведения детей с ВЗК должен стать мониторинг КЖ, связанного со здоровьем, поскольку для этой категории больных характерно значительное его снижение относительно соответствующих показателей у здоровых сверстников.

analyses - part II. Inflamm. Bowel Dis. 2018; 24(5): 966-76. DOI: 10.1093/ibd/izy015

3. Lemberg D.A., Day A.S. Crohn disease and ulcerative colitis in children: an update for 2014. J. Paediatr. Child Health. 2015; 51(3): 266-70. DOI: 10.1111/jpc.12685

4. Goldstein-Leever A., Bass J.A., Goyal A. et al. Health-related quality of life predicts psychology referral in youth with inflammatory 
bowel disease. J. Pediatr. Nurs. 2019; 47: 73-7. DOI: 10.1016/j. pedn.2019.04.016

5. Gasparetto M., Guariso G. Crohn's disease and growth deficiency in children and adolescents. World J. Gastroenterol. 2014; 20(37): 13219-33. DOI: 10.3748/wjg.v20.i37.13219

6. Werkstetter K.J., Ullrich J., Schatz S.B. et al. Lean body mass, physical activity and quality of life in paediatric patients with inflammatory bowel disease and in healthy controls. J. Crohns Colitis. 2012; 6(6): 665-73. DOI: 10.1016/j.crohns.2011.11.017

7. Cioffi I., Imperatore N., Di Vincenzo 0. et al. Association between health-related quality of life and nutritional status in adult patients with Crohn's disease. Nutrients. 2020; 12(3): 746. D0I: 10.3390/ nu12030746

8. Gallo J., Grant A., Otley A.R. et al. Do parents and children agree? Quality-of-life assessment of children with inflammatory bowel disease and their parents. J. Pediatr. Gastroenterol. Nutr. 2014; 58(4): 481-5. DOI: 10.1097/MPG.0000000000000236

9. Sehgal P., Ungaro R.C., Foltz C. et al. High levels of psychological resilience associated with less disease activity, better quality of life, and fewer surgeries in inflammatory bowel disease. Inflamm. Bowel Dis. 2020; izaa196. D0I: 10.1093/ibd/izaa196

10. Reigada L.C., Hoogendoorn C.J., Walsh L.C. et al. Anxiety symptoms and disease severity in children and adolescents with Crohn disease. J. Pediatr. Gastroenterol. Nutr. 2015; 60(1): 30-5. DOI: 10.1097/ MPG.0000000000000552

11. Marinelli C., Savarino E., Inferrera M. et al. Factors influencing disability and quality of life during treatment: a cross-sectional study on IBD patients. Gastroenterol. Res. Pract. 2019; 2019: 5354320. DOI: $10.1155 / 2019 / 5354320$

12. Денисова Р.В., Алексеева Е.И., Альбицкий В.Ю. и др. Надежность, валидность и чувствительность русских версий опросников PedsQL Generic Core Scale u PedsQL Rheumatology Module. Вопросы современной педиатрии. 2009; 8(1): 30-40. [Denisova R.V., Alexeeva E.I., Al'bitsky V.Yu. et al. Reliability, validity and sensitivity of Russian versions of PedsQL Generic Core Scale and PedsQL Rheumatology Module Questionnaires. Current Pediatrics. 2009; 8(1): 30-40. (in Russian)]

13. Корниенко Е.А., Потапов А.С., Бельмер С.В. и др. Рекомендации по диагностике и лечению болезни Крона у детей (проект). Вопросы детской диетологии. 2017; 15(5): 50-77. [Kornienko E.A., Potapov A.S., Belmer S.V. et al. Guidelines for diagnosis and treatment of Crohn"s disease in children (project). Pediatric Nutrition. 2017; 15(5): 50-77. (in Russian)]. DOI: 10.20953/1727-5784-2017-5-50-77

14. Ojeda Z.C., Cofré D.C. Health related quality of life in pediatric patients with inflammatory bowel disease. Rev. Chil. Pediatr. 2018; 89(2): 196-201. D0I: 10.4067/S0370-41062018000200196

15. van der Have M., Brakenhoff L.K., van Erp S.J. et al. Back/joint pain illness perceptions and coping are important predictors of quality of life and work productivity in patients with inflammatory bowel disease: a 12-month longitudinal study. J. Crohns Colitis. 2015; 9(3): 276-83. DOI: 10.1093/ecco-jcc/jju025

16. Bramuzzo M., De Carlo C., Arrigo S. et al. Parental psychological factors and quality of life of children with inflammatory bowel disease. J. Pediatr. Gastroenterol. Nutr. 2020; 70(2): 211-17. DOI: 10.1097/MPG.0000000000002548 D

\title{
ЧИТАЙТЕ на journaldoctor.ru
}

\author{
болезнь Крона
}

Статьи схожей тематики в выпусках «Доктор.Ру» Гастроэнтерология и «Доктор.Ру» Педиатрия:

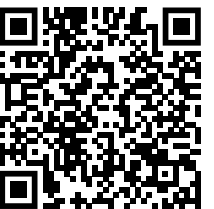

Поддубный И.В., Щербакова О.В., Козлов М.Ю., Исмаилов М.У., Трунов В.О., Алиева Э.И., Манукян С.Р. Лечение осложненной болезни Крона у подростков: обзор литературы и собственных наблюдений // Доктор.Ру. 2017. № 12 (141). С. 56-61.

Горелов А.В., Яблокова Е.А., Борисова Е.В., Полотнянко Е.Ю. и др. Особенности терапии болезни Крона осложненного течения у девочки 17 лет // Доктор.Ру. 2016. № 6 (123). С. 28-31.

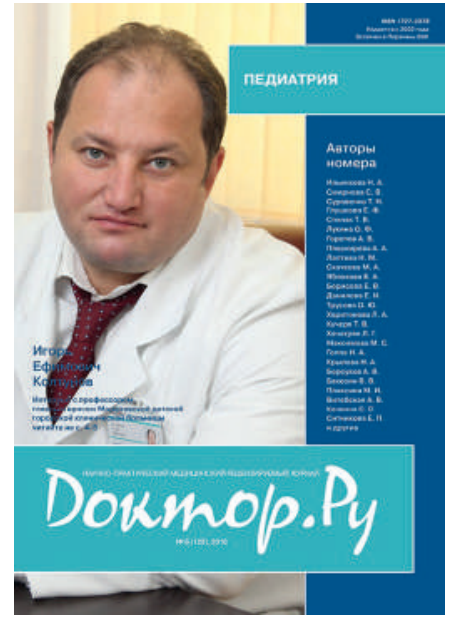

